\title{
Multiwave Monitoring of the Nuclei of Seyfert Galaxies and Quasars at Several Telescopes of FSU and European Countries in the Frame of an INTAS Program.
}

\author{
N.G.Bochkarev \\ Sternberg Astronomical Institute Universitetskij Prosp., 13, 119899 \\ Moscow, Russia boch@astronomy.msk su \\ A.I.Shapovalova, A.N.Burenkov, V.V.Vlasyuk \\ Special Astrophysical Observatory of RAS 357147 Nizhnij Arkhyz, \\ Karachaevo-Cherkessia, Russia
}

\begin{abstract}
Several groups of astronomers in FSU (Former Soviet Union) and European countries have started, in 1998, a cooperative spectral and broad-band optical and UV-monitoring of the nuclei of selected Seyfert galaxies and quasars (10 objects). The monitoring is to be continued until Oct. 30, 2000. The spectral optical monitoring with CCD-cameras in the spectral range $\lambda \lambda 4000-8000 \AA \AA$ is being carried out at two observatories: SAO RAS, Russia (6-m and 1-m telescopes) and at Kazakhstan (70-cm telescope). The resolution is about $3-10 \AA$, the $\mathrm{S} / \mathrm{N}$ ratio is $\approx 50-100$. The broad-band optical monitoring with BVRI filters (with a $1 \%$ accuracy at the R-band) is being done at 5 observatories. NLR and ENLR in monitored galaxies will also be investigated.
\end{abstract}

\section{Introduction}

A program of AGN spectral monitoring based on theoretical analyses (Blandford and McKee, 1982; Bochkarev and Antokhin, 1982; Antokhin and Bochkarev, 1983) was initiated at the 6-m telescope observatory (SAO RAS). The first test observations were carried through in 1983; regular observations started in 1986 continue up to now (see e.g. Bochkarev et al., 1990, 1991). Observations with one single telescope, however, are not sufficient for determination of AGN physical characteristics. Therefore we joined the global "AGN Watch" program (see, e.g., Peterson et al., 1991) at its starting time in 1988 and continue to work in its frame. To increase Russian/the Former Soviet Union (FSU) input to the "AGN Watch" we are starting now, in collaboration with colleagues from West-European countries, a FSU-European program supported by INTAS .

\section{Claimed objectives}

The aim of this program is to determine the fundamental characteristics of the physical structure and the kinematics of the broad (BLR) and narrow (NLR and ENLR) emission line regions in a representative sample of Seyfert galaxies. 
Specific objectives are to:

1) determine the BLR structure by application of cross-corelation methods;

2) determine the long-term stability of the BLR;

3) determine the physical state of the BLR by modeling the observed spectra;

4) determine the spatial structure, the physical state and the ionization state of the NLR and ENLR;

5) compare the line and continuum properties of Seyfert galaxies of different types covering a large range in nuclear luminosity.

\section{Spectral and broadband monitoring}

Nuclear spectra of the selected galaxies will be taken with three telescopes using CCD: with the $6-\mathrm{m}$ and $1-\mathrm{m}$ telescopes of SAO RAS ; with the $70 \mathrm{~cm}$ telescope in Kazakhstan; spectral range $\lambda \lambda 4000-8000 \AA \AA$, spectral resolution $3-10 \AA$ and signal-to-noise ratio of $50-100$ in the continuum.

Photometric observations with an accuracy of about $1 \%$ in $\mathrm{R}$ filter will be carried out at 5 observatories: SAO RAS with CCD in BVR filters on 1-m and $60-\mathrm{cm}$ telescopes; Landessternwarte, Heidelberg, with the $70-\mathrm{cm}$ telescope in BVRI filters; Crimea (Ukraine) with the $60-\mathrm{cm}$ telescope equipped with an electro-photometer and a simple CCD-camera in UBVR filters; Lesniki (near Kiev, Ukraine), with a $70-\mathrm{cm}$ or $50-\mathrm{cm}$ telescope with an electro-photometer in UBVR filters, after equipping it with an ST-6 CCD-camera; Maidanak Mountain Observatory (Republic of Uzbekistan), with a $60-\mathrm{cm}$ telescope with an electrophotometer in the UBVR filters.

\section{Spectrophotometry of the NLR}

Spectra of the NLR and ENLR will be obtained at the prime focus of the 6-m telescope of SAO RAS using the multi-pupil field spectrograph (MPFS) + CCD in the range $\lambda \lambda 4000-8000 \AA \AA$, with a resolution of about $4-6 \AA$, and a signal-tonoise ratio of 50-100 in the nuclear region and 10 in the off- nuclear regions. Blocks of 16x16 micro-lenses will be used in the spectrograph MPFS, and it will be possible to register simultaneously 256 spectra of the galaxy over a region of $16 \times 16$ arcsec.

\section{Data analysis}

Time lags of prominent emission lines will be determined by cross- correlation methods probably by two independent teams. We plan to produce images in lines and continuum, maps of velocity fields, relative intensities, line FWHI distribution etc. The archival spectra from the 6-meter telescope, from Kazakhstan, and from Crimea obtained in 1976-1996 for NGC 4151, 3516, 7469, will be used to study the long-term evolution of the BLR in these galaxies. Photometric data for the same period will be used also. The data obtained will be interpreted in the framework of photoionization models. Interpretation and theoretical re- 
searches will be done by five teams: in France, GB, Germany, Kazakhstan, and Russia.

This research was supported by INTAS-grant $96-328$ and by the Russian Basic Research Foundation through grant 97-02-17625.

\section{References}

Antokhin, I.I., Bochkarev, N.G., 1983, Astron.Zh., v.60, p.448.

Blandford, R.D., McKee, C.F., 1982, Astrophys.J., v.255, p.419.

Bochkarev, N.G., Antokhin, I.I., 1982, Astron.Tsirc., No. 1228, p.1

Bochkarev, N.G., Shapovalova, A.I., Zhekov, S.A., 1990, Astron.J., v.100, p.1799.

Bochkarev, N.G., Shapovalova, A.I., Zhekov, S.A., 1991, Astron.J., v.102, p.1278.

Peterson, B.M., et al., 1991, Astrophys.J., v. 368, p.119. 